\title{
LINEAR STABILITY OF TRIANGULAR EQUILIBRIUM POINTS IN THE GENERALIZED PHOTOGRAVITATIONAL RESTRICTED THREE BODY PROBLEM WITH POYNTING-ROBERTSON DRAG
}

\author{
B. ISHWAR ${ }^{A}$ AND B.S. KUSHVAH ${ }^{B}$ \\ $A$ P.I DST PROJECT, $B$ JRF DST PROJECT \\ UNIVERSITY DEPARTMENT OF MATHEMATICS \\ B.R.A. BIHAR UNIVERSITY MUZAFFARPUR-842001 \\ BIHAR (INDIA) \\ EMAIL:ISHWAR_BHOLA@HOTMAIL.COM, BSKUSH@HOTMAIL.COM
}

(Received 27 October 2005)

\begin{abstract}
In this paper we have examined the linear stability of triangular equilibrium points in the generalised photogravitational restricted three body problem with Poynting-Robertson drag. We have found the position of triangular equilibrium points of our problem. The problem is generalised in the sense that smaller primary is supposed to be an oblate spheroid. The bigger primary is considered as radiating. The equations of motion are affected by radiation pressure force, oblateness and P-R drag. All classical results involving photogravitational and oblateness in restricted three body problem may be verified from this result. With the help of characteristic equation, we discussed the stability. Finally we conclude that triangular equilibrium points are unstable.
\end{abstract}

\section{AMS Classification:70F15}

Keywords: Stability/Triangular Points/Generalized Photogravitational/P-R drag/RTBP.

\section{INTRODUCTION}

We study the motion of three finite bodies in the three body problem. The problem is restricted in the sense that one of the three masses is taken to be so small that the gravitational effect on the other masses by third mass is negligible. The smaller body is known as infinitesimal mass and remaining two massive bodies as finite masses. The classical restricted three body problem is generalised to include the force of radiation pressure, the Poynting-Robertson effect and oblateness effect. J.H. Poynting(1903) considered the effect of the absorption and subsequent re-emission of sunlight by small isolated particles in the solar system. His work was later modified by H.P. Robertson(1937) who used a precise relativistic treatments of the first order in the ratio of the velocity of the particle to that of light. The motion of an infinitesimal body near one of the Lagrangian points is said to be stable if given a very small displacement and small velocity, the body oscillates for a considerable time around the point. If it departs from the point as the time increases, the motion is said to be unstable. Mathematically, we express that the stability of motion means bounded displacement and velocity with bounded functions of time in the neighborhood of the equilibrium points.

If a satellite flies high enough above the Earth and is large enough in size, but at the same time has a sufficiently small mass, then the pressure of the Sun's light rays (known as radiation pressure ) has a very strong effect on its motion. The distance of the satellite to the Sun practically does not change, and so the magnitude of the radiation pressure force is practically constant. The Poynting-Robertson (P-R) effect is the most important nongravitational and nondisipative interplanetary dust particles. The P-R effect may be significant in dealing with other problems in astrophysics.

JOURNAL OF DYNAMICAL SYSTEMS \& GEOMETRIC THEORIES

VOL. 4, NUMBER 1 (2006) 0-0.

(C)TARU PUBLICATIONS 
The effect of radiation pressure and P-R drag in the restricted three body problem has been studied by Colombo et al.(1966), Chernikov Yu.A.(1970) and Schuerman(1980) discussed the position as well as the stability of the Lagrangian equilibrium points when radiation pressure, P-R drag force are included. Murray C.D.(1994) systematically discussed the dynamical effect of general drag in the planar circular restricted three body problem. Liou J.C. et al.(1995) examined the effect of radiation pressure, P-R drag and solar wind drag in the restricted three body problem. The classical three body problem has five Lagrangian points. Their location and stability properties are well known. The three collinear points $L_{1}, L_{2}, L_{3}$ lies on the line joining the two primaries, are linearly unstable. The two Lagrangian points $L_{4}, L_{5}$ lie in the orbit of the test particle which are symmetrical about the line joining the primaries, are linearly stable with the condition that the mass parameter $\mu$ to be less than .0385 . Hence, we are interested to discuss the linear stability of triangular equilibrium points in generalised photogravitational restricted three body problem with PoyntingRobertson drag. After Newton solved the problem of the orbit of single planet around the Sun, the natural next challenge was to find the solution of two planets orbiting the Sun(i.e. three body problem ). Many of the best minds in Mathematics and Physics worked on this problem in the last century. Three-Body problem is a continuous source of study, since the discovery of its non-integrability due to Poincare(1892). Regular and chaotic motion have been widely investigated with any kind of tools, from analytical result to numerical explorations. The restricted three-body problem is a modified three-body problem when one of the three masses is set to zero(infinitesimal mass ).

The restricted three body model, Earth-Moon and Spacecraft is insoluble, although specific solutions exist, like the ones in which the spacecraft is positioned at one of the Lagrangian points. There are many periodic orbits in the restricted three body problem. One of the most famous, discovered by Lagrange, is formed by an equilateral triangle. In "Earth-Moon-Space Station model", if the Moon is not too massive, this orbit is thought to be stable. If the space station is pushed a bit to one side (in position or velocity), it's supposed to make small oscillations around this orbit. The two kinds of triangular points are called $L_{4}$ and $L_{5}$ points.

There is a group of enthusiasts who want to setup a colony at $L_{5}$ point of the Earth-Moon system. As already noted, because $L_{4}$ and $L_{5}$ are the stable points of equilibrium, they have been proposed for sites of large self-contained "Space colonies", an idea developed and advocated by the late Geraid O' Neill (1974). The three body problem have an interesting application for artificial satellites and future space colonization. Triangular points of the Sun-Jupiter or Sun-Earth system would be convenient sites to locate future space colonies. Such colonies would presumably mine asteroids for at least some of their raw materials. Present day technology permits the construction of spacecraft whose value of $\beta$ (ratio of radiation force and gravitation force) can be made or varied in the range $0<\beta<1$. (A spacecraft with $\beta>1$ has been considered by NASA as a "Sailing" vehicle for chasing Halley's comet [Friedman 1976]). A Space Colony with a solar facing adjustable "sail" could thus "park" (i.e. remain stationary with respect to the Sun and a planet without expending large amounts of energy) not only at the classical positions but at any heleocentricdistance out to that of the planet.

Thus we have included the purpose of the work and its significance in the field of Celestial Mechanics and Dynamical Astronomy. Application of results to realistic actual problem is obvious. $L_{4}$ and $L_{5}$ points became unstable due to P-R drag which is very remarkable and important, where as they are stable in classical result. 


\section{Location of Triangular Equilibrium Points}

Equations of motion are,

$$
\begin{array}{cc}
\ddot{x}-2 n \dot{y}=U_{x}, \quad \text { where, } \quad U_{x}=\frac{\partial U_{1}}{\partial x}-\frac{W_{1} N_{1}}{r_{1}^{2}} \\
\ddot{y}+2 n \dot{x}=U_{y}, \quad U_{y}=\frac{\partial U_{1}}{\partial y}-\frac{W_{1} N_{2}}{r_{1}^{2}} \\
\ddot{z}=U z, \quad U_{z}=\frac{\partial U_{1}}{\partial z}-\frac{W_{1} N_{3}}{r_{1}^{2}} \\
U_{1}=\frac{n^{2}\left(x^{2}+y^{2}\right)}{2}+\frac{(1-\mu) q_{1}}{r_{1}}+\frac{\mu}{r_{2}}+\frac{\mu A_{2}}{2 r_{2}^{3}} \\
r_{1}^{2}=(x+\mu)^{2}+y^{2}, \quad r_{2}^{2}=(x+\mu-1)^{2}+y^{2}, \quad n^{2}=1+\frac{3}{2} A_{2}, \\
N_{1}=\frac{(x+\mu) N}{r_{1}^{2}}+\dot{x}-n y, \quad N_{2}=\frac{y N}{r_{1}^{2}}+\dot{y}+n(x+\mu), \quad N_{3}=\frac{z N}{r_{1}^{2}}+\dot{z}, \\
N=(x+\mu) \dot{x}+y \dot{y}+z \dot{z}
\end{array}
$$

$A_{2}=\frac{r_{e}^{2}-r_{p}^{2}}{5 r^{2}}$ be the oblateness coefficient, $r_{e}$ and $r_{p}$ be the equatorial and polar radii respectively $r$ be the distance between primaries, $q=\left(1-\frac{F_{p}}{F_{g}}\right)$ be the mass reduction factor expressed in terms of the particle's radius $a$, density $\rho$ and radiation pressure efficiency factor $\chi$ (in the C.G.S.system) i.e., $q=1-\frac{5.6 \times 10^{-5} \chi}{a \rho}$. Assumption $q=$ constant is equivalent to neglecting fluctuation in the beam of solar radiation and the effect of solar radiation, the effect of the planet's shadow, obviously $q \leq 1$. Triangular equilibrium points are given by $U_{x}=0, U_{y}=0, z=0, y \neq 0$, then we have

$$
\begin{aligned}
& x_{*}=x_{0}\left\{1-\frac{n W_{1}\left[(1-\mu)\left(1+\frac{5}{2} A_{2}\right)+\mu\left(1-\frac{A_{2}}{2}\right) \frac{\delta^{2}}{2}\right]}{3 \mu(1-\mu) y_{0} x_{0}}-\frac{\delta^{2}}{2} \frac{A_{2}}{x_{0}}\right\} \\
& y_{*}=y_{0}\left\{1-\frac{n W_{1} \delta^{2}\left[2 \mu-1-\mu\left(1-\frac{3 A_{2}}{2}\right) \frac{\delta^{2}}{2}+7(1-\mu) \frac{A_{2}}{2}\right]}{3 \mu(1-\mu) y_{0}^{3}}-\frac{\delta^{2}\left(1-\frac{\delta^{2}}{2}\right) A_{2}}{y_{0}^{2}}\right\}^{1 / 2}
\end{aligned}
$$

where $x_{0}=\frac{\delta^{2}}{2}-\mu, y_{0}= \pm \delta\left(1-\frac{\delta^{2}}{4}\right)^{1 / 2}$ and $\delta=q_{1}^{1 / 3}$

where subscript $*$ indicate the value at the equilibrium points. Eq. (5) and (6) are valid for $W_{1} \ll 1$, $A_{2} \ll 1$.

\section{Stability of Triangular Equilibrium Points}

We suppose $\alpha=A_{1} e^{\lambda t}, \beta=B_{1} e^{\lambda t}, \gamma=C_{1} e^{\lambda t}$ be the small displacements from Lagrangian points $\left(x_{*}, y_{*}, z_{*}\right)$ and $A_{1}, B_{1}, C_{1}, \lambda$ are parameters, $x=x_{*}+\alpha, \quad y=y_{*}+\beta \quad$ and $\quad z=z_{*}+\gamma$. Therefore the equations of perturbed motion corresponding to the system of Eq.(1),(2),(3) may be written as,

$$
\begin{aligned}
\ddot{\alpha}-2 n \dot{\beta} & =U_{x}^{0}+\alpha U_{x x}^{0}+\beta U_{x y}^{0}+\gamma U_{x z}^{0}+\dot{\alpha} U_{x \dot{x}}^{0}+\dot{\beta} U_{x \dot{y}}^{0}+\dot{\gamma} U_{x \dot{z}}^{0} \\
\ddot{\beta}+2 n \dot{\alpha} & =U_{y}^{0}+\alpha U_{y x}^{0}+\beta U_{y y}^{0}+\gamma U_{y z}^{0}+\dot{\alpha} U_{y \dot{x}}^{0}+\dot{\beta} U_{y \dot{y}}^{0}+\dot{\gamma} U_{y \dot{z}}^{0} \\
\ddot{\gamma} & =U_{z}^{0}+\alpha U_{z x}^{0}+\beta U_{z y}^{0}+\gamma U_{z z}^{0}+\dot{\alpha} U_{z \dot{x}}^{0}+\dot{\beta} U_{z \dot{y}}^{0}+\dot{\gamma} U_{z \dot{z}}^{0} \\
\text { At } \quad\left(x_{*}, y_{*}, z_{*}\right) \quad U_{x}^{0}= & U_{y}^{0}=U_{z}^{0}=U_{x z}^{0}=U_{y z}^{0}=U_{z x}^{0}=U_{z y}^{0}=U_{x \dot{z}}^{0}=U_{y \dot{z}}^{0}=U_{z \dot{x}}^{0}=U_{y \dot{z}}^{0}=0
\end{aligned}
$$

Above system of equations can be written as,

$$
\begin{aligned}
&\left(\lambda^{2}-\lambda U_{x \dot{x}}^{0}-U_{x x}^{0}\right)+\left[-\left(2 n+U_{x \dot{y}}^{0}\right) \lambda-U_{x y}^{0}\right] B_{1}=0 \\
& {\left[\left(2 n-U_{y \dot{x}}^{0}\right) \lambda-U_{y x}^{0}\right] A_{1}+\left(\lambda^{2}-\lambda U_{y \dot{y}}^{0}-U_{y y}^{0}\right) B_{1}=0 } \\
&\left(\lambda^{2}-\lambda U_{z \dot{z}}^{0}-U_{z z}^{0}\right) C_{1}=0
\end{aligned}
$$


Eq.(12) gives us, $\lambda=\frac{U_{z \dot{z}}^{0} \pm \sqrt{\left(U_{z \dot{z}}^{0}\right)^{2}+4 U_{z z}^{0}}}{2}$. From Eq.(3)

$$
\begin{gathered}
U_{z z}^{0}=-f, \quad U_{z \dot{z}}^{0}=-\frac{W_{1}}{r_{1}^{2}}, \quad \text { where, } \quad f=\frac{(1-\mu) q_{1}}{r_{1}^{3}}+\frac{\mu}{r_{2}^{3}}\left(1+\frac{3}{2} \frac{A_{2}}{r_{2}^{2}}\right) \\
\lambda=\frac{1}{2}\left\{-\frac{W_{1}}{r_{1}^{2}} \pm \sqrt{\frac{W_{1}^{2}}{r_{1}^{4}}-4 f}\right\} \quad \text { as } \quad W_{1} \geq 0 \quad \text { and } \quad f>\frac{W_{1}^{2}}{4 r_{1}^{4}}
\end{gathered}
$$

Since $\lambda$ consists always negative real part, so motion is asymptotically stable in $z$ direction. Now Eq.(10) and (11) has singular solution if,

$$
\begin{gathered}
\left|\begin{array}{cc}
\lambda^{2}-\lambda U_{x \dot{x}}^{0}-U_{x x}^{0} & -\left(2 n+U_{x \dot{y}}^{0}\right) \lambda-U_{x y}^{0} \\
\left(2 n-U_{y \dot{x}}^{0}\right) \lambda-U_{y x}^{0} & \lambda^{2}-\lambda U_{y \dot{y}}^{0}-U_{y y}^{0}
\end{array}\right|=0 \\
\Rightarrow \quad \lambda^{4}+a \lambda^{3}+b \lambda^{2}+c \lambda+d=0
\end{gathered}
$$

At the equilibrium points the system of Eq.(1),(2) gives

$$
\begin{aligned}
a & =3 \frac{W_{1}}{r_{1 *}^{2}}, \quad b=2 n^{2}-f_{*}-\frac{3 \mu A_{2}}{r_{2 *}^{5}}+\frac{2 W_{1}^{2}}{r_{1 *}^{4}}=b_{0}+b_{1} \quad b_{0}=2 n^{2}, b_{1}=-f_{*}-\frac{3 \mu A_{2}}{r_{2 *}^{5}}+\frac{2 W_{1}^{2}}{r_{1 *}^{4}} \\
c & =-a\left\{n^{2}+\frac{\mu A_{2}}{r_{2 *}^{5}}+\frac{\mu}{r_{1 *}^{2} r_{2 *}^{5}}\left(1+\frac{5 A_{2}}{2 r_{2 *}^{2}}\right) y_{*}^{2}\right\} \\
d & =\left(n^{2}-f_{*}\right)\left[n^{2}+2 f_{*}-\frac{3 \mu A_{2}}{r_{2 *}^{5}}\right]+\frac{9 \mu(1-\mu) q_{1}}{r_{1 *}^{5} r_{2 *}^{5}}\left(1+\frac{5 A_{2}}{2 r_{2 *}^{2}}\right) y_{*}^{2} \\
& -\frac{6 n W_{1}}{r_{1 *}^{4} r_{2 *}^{5}}\left(1+\frac{5 A_{2}}{2 r_{2 *}^{2}}\right)\left\{\left(x_{*}+\mu\right)^{2}-\left(x_{*}+\mu\right)+y_{*}^{2}\right\}+\frac{n^{2} W_{1}^{2}}{r_{1 *}^{4}}
\end{aligned}
$$

where

$$
r_{1 *}=q_{1}^{1 / 3}\left\{1-\frac{n W_{1}}{6(1-\mu) y_{0}}-\frac{A_{2}}{2}\right\}, \quad r_{2 *}=1+\frac{n W_{1}}{3 \mu y_{0}}\left(1-\frac{5}{2} A_{2}\right)
$$

Let $q=1-\epsilon$ with $\epsilon>0$ very small real quantity then

$$
f_{*}=1-\frac{n W_{1}(9+2 \epsilon)\left(4-3 A_{2}\right)}{18 \sqrt{3}}+3 \frac{(1-\mu) A_{2}}{2}
$$

We can write the four roots of the classical characteristic equation as,

$$
\lambda_{j}= \pm z i, \quad \text { where, } \quad z^{2}=\frac{1}{2}\left\{1 \mp[1-27 \mu(1-\mu)]^{1 / 2}\right\}, \quad j=1,2,3,4 .
$$

Due to P-R drag and oblateness, we assume the solution of the characteristic Eq.(14) of the form

$$
\lambda=\lambda_{j}\left(1+e_{1}+e_{2} i\right)= \pm\left[-e_{2}+\left(1+e_{1}\right) i\right] z
$$

where $e_{1}, e_{2}$ are small real quantities. We have in first order approximation

$$
\lambda^{2}=\left[-\left(1+2 e_{1}\right)-2 e_{2} i\right] z^{2}, \quad \lambda^{3}= \pm\left[3 e_{2}-\left(1+3 e_{1}\right) i\right] z^{3}, \quad \lambda^{4}=\left[\left(1+4 e_{1}\right)+4 e_{2} i\right] z^{4}
$$

Substituting these values in characteristic Eq.(14) neglecting products of $e_{1}$ or $e_{2}$ with $a, b_{1}, c$, we get

$$
\begin{aligned}
& e_{1}=\frac{-d+b_{0} z^{2}-z^{4}}{2 z^{2}\left(2 z^{2}-b_{0}\right)} \\
& e_{2}=\frac{\mp c z \pm a z^{3}}{2 z^{2}\left(2 z^{2}-b_{0}\right)}
\end{aligned}
$$

If $e_{2} \neq 0$, then the resulting motion of particle displaced from equilibrium points is asymptotically stable only when all the real parts of $\lambda$ are negative. For stability, we require $\operatorname{Re}(\lambda)<0$. Rate of growth or decay 
is determined by the largest real part of the roots of the characteristic equation, where $\operatorname{Re}(\lambda)$ denotes the real parts of $\lambda$ and given by

$$
\operatorname{Re}(\lambda)=\frac{c-a z^{2}}{2\left(2 z^{2}-1\right)}
$$

we have,

$$
z^{2}=\frac{1}{2}\left\{1 \mp[1-27 \mu(1-\mu)]^{1 / 2}\right\}
$$

Taking positive sign ,

$$
z^{2}=1-\frac{27}{4} \mu(1-\mu)
$$

Consider

$$
\begin{gathered}
\operatorname{Re}(\lambda)<0 \text { then } \quad\left\{c-a\left[1-\frac{27}{4} \mu(1-\mu)\right]\right\}\left\{1-\frac{27}{2} \mu(1-\mu)\right\}^{-1}<0 \\
\Rightarrow \quad c+\frac{27}{4} \mu(1-\mu)(2 c-a)<a
\end{gathered}
$$

Taking negative sign,

From above inequalities we get,

$$
\begin{aligned}
\operatorname{Re}(\lambda) & =-\left[c-a \frac{27}{4} \mu(1-\mu)\right]\left[1-\frac{27}{2} \mu(1-\mu)\right]^{-1}<0 \\
& \Rightarrow 0<c+\frac{27}{4} \mu(1-\mu)(2 c-a)
\end{aligned}
$$

$$
\begin{aligned}
& 0<c+\frac{27}{4} \mu(1-\mu)(2 c-a)<a \\
& 0<c<a \text { as } \mu \rightarrow 0 \quad \text { Murray C.D.(1994) }
\end{aligned}
$$

Inequality (26) is necessary condition for stability of triangular equilibrium points $L_{4}$ and $L_{5}$.

\section{Conclusion}

(i) When $W_{1}=0$, then

$$
\begin{aligned}
& a=0, c=0, b=n^{2}-3 \mu A_{2}, \\
& d=9 \mu(1-\mu)\left(1+\frac{5}{2} A_{2}\right)\left(1-\frac{q_{1}^{2 / 3}}{4 n^{4}}\right) n^{2}
\end{aligned}
$$

Equation (14) gives us,

$$
\begin{gathered}
\lambda^{2}=\frac{-b \pm\left(b^{2}-4 d\right)^{1 / 2}}{2} \text { For stable motion } \quad b^{2}>4 d \\
\text { i.e., } \quad\left(n^{2}-3 \mu A_{2}\right)^{2}>36 \mu(1-\mu)\left(1+\frac{5}{2} A_{2}\right)\left(1-\frac{q_{1}^{2 / 3}}{4 n^{4}}\right) n^{2}
\end{gathered}
$$

(ii) For classical case $A_{2}=0, q_{1}=1, W_{1}=0, n=1$. Then from inequality (26) we have

$$
1>27 \mu(1-\mu) \quad \Rightarrow \quad \mu<.0385
$$

(iii) When $W_{1} \neq 0, A_{2}=0, n=1$. Then

$$
\begin{aligned}
& a=3 \frac{W_{1}}{r_{1 *}^{2}}, b=2-f_{*}+\frac{2 W_{1}^{2}}{r_{1_{*}}^{4}}, c=-a\left\{1+\frac{\mu y_{*}^{2}}{r_{1_{*}}^{2} r_{1_{*}}^{5}}\right\} \\
& d=\left(1-f_{*}\right)\left(1+2 f_{*}\right)+\frac{9 \mu(1-\mu) q_{1}}{r_{1_{*}}^{2} r_{1_{*}}^{5}} y_{*}^{2}-\frac{6 W_{1}}{r_{1_{*}}^{4} r_{2_{*}}^{5}}\left[\left(x_{*}+\mu\right)^{2}-\left(x_{*}+\mu\right)+y_{*}^{2}\right]+\frac{W_{1}^{2}}{r_{1_{*}}^{4}}
\end{aligned}
$$

as in Chernikove Yu.A.(1970) 
If we consider the P-R drag force then $c<0$. This dose not satisfy the necessary condition of stability. Hence motion is unstable in the linear sense.

Acknowledgment: We are thankful to D.S.T., Government of India, New Delhi for sanctioning a project DST/MS/140/2K dated 02/01/2004 on this topic.

\section{REFERENCES}

[1] Chernikov Yu.A.(1970): The photogravitational restricted three body problem. Sov. Astr. AJ. 14(1), $176-181$.

[2] Colombo G.D. Lautman and Shapiru I.I.(1996): The Earth's dust belt:Fact or fiction? 2.Gravitational focusing and Jacobi capture. J. Geophys. Res. 71, 5705-5717.

[3] Friedman L. D. (1975): In Proceedings of the Shuttle-Based Cometary Science Workshop.ed. G.A. Garyand K. S. Chfton(Huntsville: NASA Marshall Space Flight Center), p. 251.

[4] Ishwar B.(1997): Nonlinear stability in the generalised restricted three body problem. Celst. Mech. and Dyn. Astron. 65, 253-289.

[5] Kushvah B.S. and Ishwar B.(2004):Triangular equilibrium points in the generalised photograviational restricted three body problem with Poynting-Robertson drag., Review Bull. Cal. Math. Soc.,12, (1 \& 2), 109-114.

[6] Liou J.C.,Zook H.A. and Jackson A.A (1995): Radiation pressure, Poynting-Robertson drag and solar wind drag in the restricted three body problem. Icarus 116, 186-201

[7] McCuskey S.W.(1963): Introduction to celestial Mechanics Addison Wesley Publishing company Inc. New York.

[8] Murray C.D.(1994): Dynamical effect of drag in the circular restricted three body problem 1. Location and stability of the Lagrangian equilibrium points . Icarus 112, 465-484.

[9] O'Neill G. K.(1974): “The colonization of space", Physics Today 27 (September), 32-40.

[10] Poincare, H.(1892): Les Methodes Nouvelles de la Mechanique Celeste,vol.1, Dover, New York,1957

[11] Ragos O. and Zafiropoulos F. A.(1995):A numerical study of the influence of the Poynting-Robertson effect on the equilibrium points of the photogravitational restricted three body problem 1. Coplanar case. Astron. Astrophys.300, 568-578.

[12] Robertson H.P.(1937): Dynamical effects of radiation in the solar system. Mon.Not. Roy. Astron. Soc. 97, 423-438.

[13] Sahoo S.K.and Ishwar B.(2000): Stability of collinear equilibrium points in the generalised photogravitational elliptical three body problem. Bull. Astron. Soc. India 28, 579-586.

[14] Schuerman D.W.(1980):The restricted three body problem including radiation pressure. Astrophys. J. 238(1), 337-342.

[15] Singh J. and Ishwar B.(1999):Stability of triangular points in generalised photogravitational restricted three body problem. Bull. Astro. Soc. India 27, 415-424.

[16] Tripathi Deepak Kumar and Ishwar B.(2005): Linear stability of triangular equilibrium points in the photogravitational restricted three body problem with Poynting-Robertson drag, News Bull. Cal. Math. Soc., 28, (1 \& 3).

[17] Wyatt S.P. and Whipple F.L.(1950): The Poynting-Robertson effect on meteor orbits. American Astr. Soc. APJ. 111, $134-141$. 